\title{
Severe Myocardial Dysfunction During Extracorporeal Membrane Oxygenation
}

\author{
By Ronald B. Hirschl, Kurt F. Heiss, and Robert H. Bartlett \\ Ann Arbor, Michigan
}

\begin{abstract}
- Of the 102 neonates with respiratory failure supported with extracorporeal membrane oxygenation (ECMO) at this institution between 1984 and 1987, 8 patients developed severe myocardial dysfunction that was noted shortly after onset of bypass. The neonates in the cardiac dysfunction group were more hypoxic laverage $\mathrm{PaO}_{2}=26 \pm 8 \mathrm{~mm} \mathrm{Hg} v$ $41 \pm 19 \mathrm{~mm} \mathrm{Hg}, P<.01$ ) in the immediate pre-ECMO period. Seventy-five percent were unstable hemodynamically 16 hypotensive, 3 bradycardic, 2 sustained cardiac arrest, 4 required epinephrine pressor support|. On ECMO, 5 of the 8 neonates developed an ischemic cardiomyopathy that lasted for less than 24 hours and resolved without therapeutic intervention. In the other 3 cases, prolonged periods of dysfunction were noted and afterload reduction through administration of tolazoline or hydralazine was beneficial. These 8 patients serve to demonstrate the reversible nature of postischemic cardiac dysfunction in patients on ECMO and in the neonatal population in general.

Copyright $\odot 1992$ by W.B. Saunders Company
\end{abstract}

INDEX WORDS: Extracorporeal membrane oxygenation (ECMO).

$\mathbf{P}$ ROLONGED extracorporeal membrane oxygenation (ECMO) support is now a standard treatment of respiratory failure in the full-term neonate. ${ }^{1,2}$ At last report, 715 neonatal ECMO cases had been performed with an overall survival of greater than $80 \% .^{3}$ At the University of Michigan we provided ECMO support to 102 neonates with severe respiratory failure between 1984 and 1987. In 8 of these cases, severe cardiac dysfunction was noted to be present shortly after onset of bypass. Cardiac electrical activity remained normal, but there was a marked decrease in pulse pressure and a concomitant decrease in cardiac contractility. Over a period of hours to days this cardiac dysfunction resolved. The purpose of this article is to discuss the phenomenon of reversible, postischemic "myocardial stun" in the neonate on ECMO and to suggest treatment options. ${ }^{4}$

From the Department of Surgery, University of Michigan Medical Center, Ann Arbor, MI.

Date accepted: November 12, 1990.

Supported in part by Grant No. HD15434 from the National Institutes of Health.

Address reprint requests to Robert $H$. Bartlett, $M D$, University of Michigan Medical Center, 1500 E Medical Center Dr, Ann Arbor, MI 48109-0331.

Copyright (1) 1992 by W.B. Saunders Company

0022-3468/92/2701-0013\$03.00/0

\section{MATERIALS AND METHODS}

Of the 102 neonates with respiratory failure who have been supported with ECMO between 1984 and 1987 at the University of Michigan, 8 developed severe left ventricular dysfunction during their ECMO course and were retrospectively identified and evaluated. The first manifestation of cardiac dysfunction on ECMO was a flat arterial line waveform. There was no change in monitored cardiac electrical activity. The possible differential diagnoses of cardiac tamponade and tension pneumothorax were ruled out with chest $\mathrm{X}$-ray and echocardiogram. Patency of the umbilical arterial line was established and the line even replaced in one case. That the patient could be hypovolemic and/or on inadvertent total bypass was considered in the differential diagnosis of a loss of pulsatile flow. On total bypass, almost all the venous return is diverted into the extracorporeal circuit. The patient is then exclusively dependent on nonpulsatile extracorporeal flow for perfusion. Hypovolemia produces a similar clinical picture because less volume is available for cardiac output and the majority of the venous return is diverted into the nonpulsatile extracorporeal circuit. Evaluation for hypovolemia or an inadvertent total bypass situation was performed by the following maneuvers: (1) the extracorporeal flow rate was decreased, which should have resulted in improvement in the pulse pressure as cardiac output increased; (2) the extracorporeal flow rate was increased, upon which the ECMO servoregulation should have stopped the pump due to insufficient venous return for the increased flow rate; and (3) volume challenges of up to $50 \mathrm{~mL}$ of albumin or blood products (approximately $15 \mathrm{~mL} / \mathrm{kg}$ ) were administered, which should have resulted in an improvement in pulse pressure. None of the expected responses was elicited and hypovolemia or a total bypass situation was excluded. Figure 1 demonstrates the cardiopulmonary profile of one child with these typical findings. The $\mathrm{SvO}_{2}$ was measured in the premembrane oxygenator ECMO circuit and was $67 \%$ after stabilization on bypass 2 hours into the ECMO course. At that time the ECMO flow rate was $350 \mathrm{~mL} / \mathrm{min}$ and the pulse pressure was 0 . With a decrease in ECMO flow rate to 250 InL/min, the pulse pressure did not increase, which would be the expected response if the initial decreased pulse pressure were secondary to achieving total bypass. Additionally, the $\mathrm{SvO}_{2}$ dropped to $46 \%$ because of the dependence of this patient on ECMO for cardiopulmonary support. Meanwhile, the $\mathrm{PaO}_{2}$ remained elevated in the 176 to 529 range because the source of almost all blood flow was the extracorporeal circuit. The ECMO circuit delivers blood with $\mathrm{PaO}_{2}$ values in the 500 range. Therefore, in this situation, because the $\mathrm{PaO}_{2}$ will remain elevated whether oxygen delivery is adequate or not, the $\mathrm{SvO}_{2}$ instead of the $\mathrm{PaO}_{2}$ must be followed in guiding requirements for ECMO flow. With increase in ECMO flow back up to $300 \mathrm{~mL} / \mathrm{min}$, note the increase in the $\mathrm{SvO}_{2}$ to $63 \%$ and then to $75 \%$ on a flow of $350 \mathrm{~mL} / \mathrm{min}$.

\section{RESULTS}

Descriptive statistics for these eight patients are presented in Table 1 . Primary diagnoses in these eight patients included meconium aspiration syndrome (3), congenital diaphragmatic hernia (3), per- 


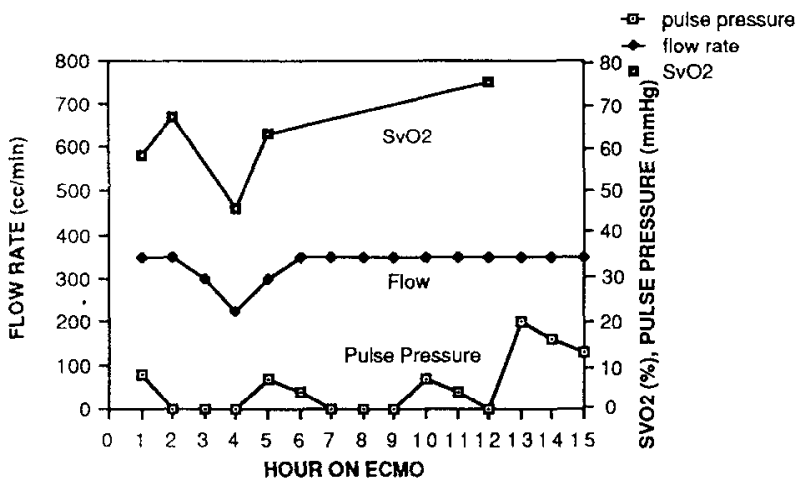

Fig 1. Cardiopulmonary profile of one child with cardiac dysfunction while on ECMO.

sistent fetal circulation (1), and $\beta$-Streptococcus sepsis (1). The average gestational age was $39 \pm 2$ weeks and the average birth weight $3.26 \pm .62 \mathrm{~kg}$. The average age at the time of institution of ECMO was $54 \pm 53$ hours and the duration of the ECMO course was $123 \pm 45$ hours. Data on the severity of the pre-ECMO cardiopulmonary insufficiency found in these patients is demonstrated in Table 1 . The oxygen index (OI) is calculated based on the following formula:

$$
\frac{\mathrm{F}_{1} \mathrm{O}_{2} \times \text { Mean Airway Pressure } \times 100}{\mathrm{PaO}_{2}}
$$

This numerical value relates the $\mathrm{F}_{\mathrm{I}} \mathrm{O}_{2}$ and postductal oxygenation to the level of ventilator pressures required to adequately ventilate the neonate. A retrospective analysis in this neonatal intensive care unit suggests that an OI greater than 40 based on three of five postductal arterial blood gases each drawn 30 minutes to 1 hour apart defines the neonate with greater than an $80 \%$ mortality. ${ }^{1}$ The last 3 oxygen indexes before onset of ECMO for each of the eight newborns were averaged and, in chronological order, were $51,71,74$. The average $\mathrm{PO}_{2}$ was $26 \pm 8 \mathrm{~mm} \mathrm{Hg}$. Six of the newborns were hypotensive, 3 were bradycardic, and 2 sustained cardiac arrest in the immediate pre-ECMO period. Four were hemodynamically compromised enough to require epinephrine support and all received dopamine and/or dobutamine, 6 for systemic hypotension and 2 in an attempt to increase systemic arterial pressures relative to pulmonary arterial pressures in order to decrease transpulmonic shunting.

Once these newborns were placed on ECMO, a decrease in pulse pressure to $<10 \mathrm{~mm} \mathrm{Hg}$ was first noted at an average of 2.5 hours into the ECMO course and lasted for an average duration of 40 hours. A pulse pressure of $<5 \mathrm{~mm} \mathrm{Hg}$ was noted at an average of 6.4 hours into the ECMO course, and the pulse pressure remained $<5 \mathrm{~mm} \mathrm{Hg}$ for an average of 12.2 hours.

Figure 2 demonstrates the pulse pressure and ECMO flow rate with time on ECMO in a child with a diaphragmatic hernia who was hypotensive, hypoxic, and on an epinephrine intravenous drip before onset of bypass support. Pulse pressure remained consistently in the 0 to $10 \mathrm{~mm} \mathrm{Hg}$ range for the first 68 hours. Attempts at improving myocardial contractility with dopamine had no evident effect on cardiac contractility. After 62 hours on ECMO, afterload reduction was attempted with intermittent intravenous hydralazine administration and a continuous amrinone intravenous drip. Shortly after onset of administration of these agents the pulse pressure improved and the neonate was weaned from bypass support without further need for pressor administration or afterload reduction.

Figure 3 depicts the course of a newborn with meconium aspiration syndrome placed on ECMO at 41 hours of age. Immediately prior to institution of bypass support, the neonate was profoundly hypoxic, hypotensive, and bradycardic requiring cardiopulmonary resuscitation and extensive pressor support. Pulse pressure diminished almost immediately after onset of ECMO support, resolving spontaneously approximately 20 hours into the ECMO course. The child did well subsequently without any evidence of cardiac sequelae.

A slightly different course is demonstrated in Fig 4. A neonate with $\beta$-Streptococcus sepsis and respiratory failure was placed on bypass at 7 days of age. Profoundly hypoxic and on dopamine in the immediate pre-ECMO period, this newborn had a moderate drop in pulse pressure to the 8 to 15 range until intravenous dopamine therapy was instituted at 34 hours into the ECMO course. Pulse pressure further decreased to minimal levels despite discontinuation of the dopamine. Afterload reduction therapy with tolazoline and hydralazine at approximately 140 hours on ECMO resulted in marked improvement in pulse pressure. Neither nitroprusside nor amrinone appeared to have any beneficial effect and dopamine, early in the course, appeared to actually lead to further deterioration in pulse pressure. Afterload reduction was continued by intermittent administration of intravenous hydralazine for the duration of the ECMO course. The child was subsequently weaned off bypass.

Overall, 3 of the 8 children had prolonged ( $>24$ hours) periods of cardiac dysfunction (Table 1). In these neonates, administration of tolazoline and/or 


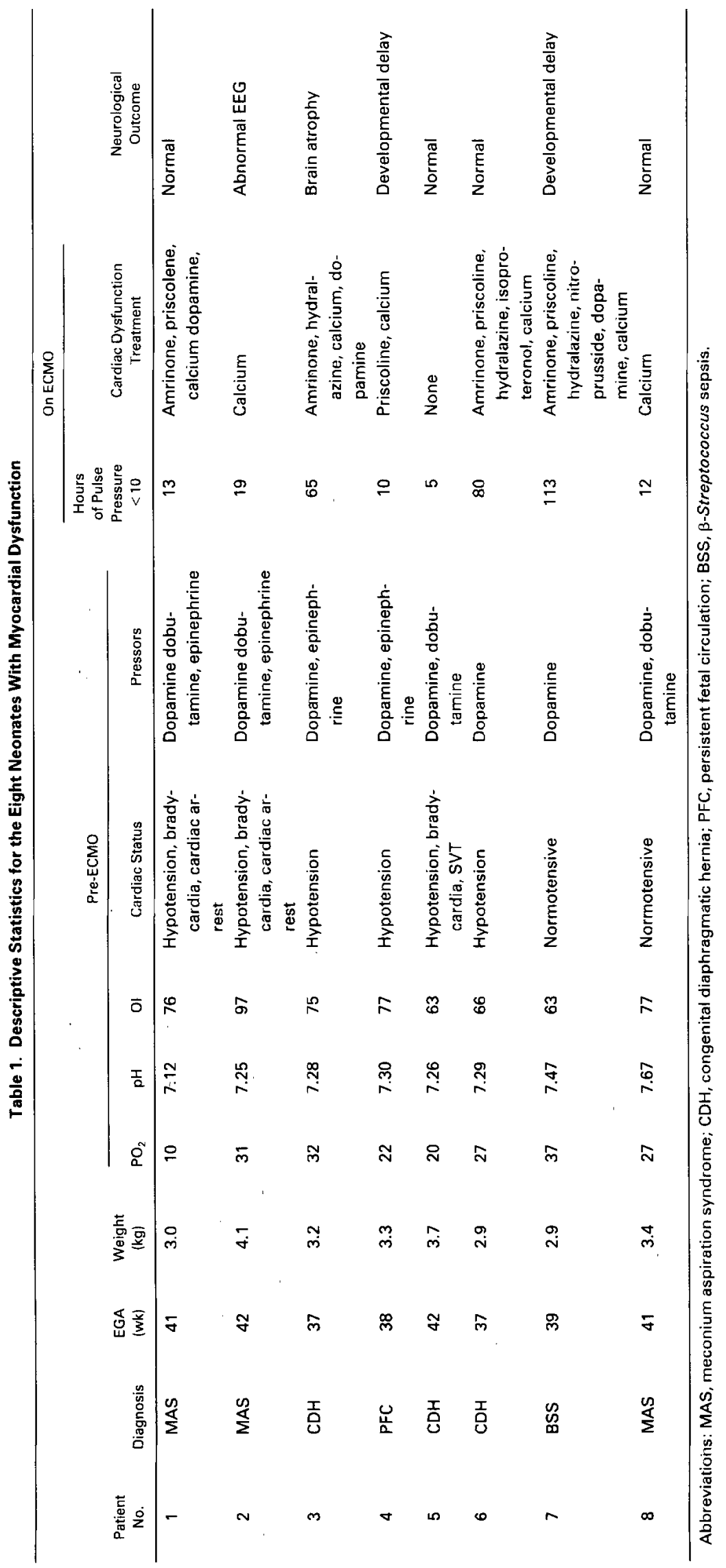




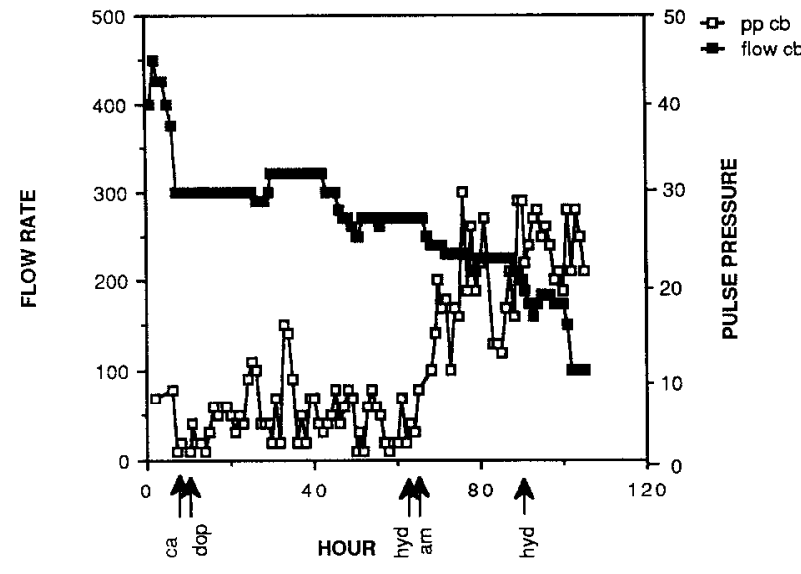

Fig 2. Pump flow rate and pulse pressure with time. A response to afterload reduction with hydralazine (hyd) and amrinone (am) administration was noted. Dop, dopamine; ca, calcium.

hydralazine directly resulted in an improvement in pulse pressure. Cardiac dysfunction lasted for less than 24 hours in the other 5 children (range, 5 to 19 hours; average, $11.8 \pm 5.1$ hours). These newborns appeared to have return of cardiac function spontaneously and unrelated to any of the pharmacological agents administered.

Echocardiographic evaluation was performed in 6 of the 8 newborns at three points in their course: (1) before institution of $\mathrm{ECMO}$; (2) during periods of cardiac dysfunction while on ECMO; and (3) after resolution of the cardiac dysfunction. Normal to slightly decreased left ventricular function (LVF) was evident in all children before onset of ECMO support. In all cases, when the pulse pressure was minimal, LVF was almost always scvercly or globally decreased. Follow-up evaluation demonstrated subsequent normal LVF in all but one child. This child had fair LVF, an improvement from the global severe decrease in LVF noted during the period of minimal pulse pressure.

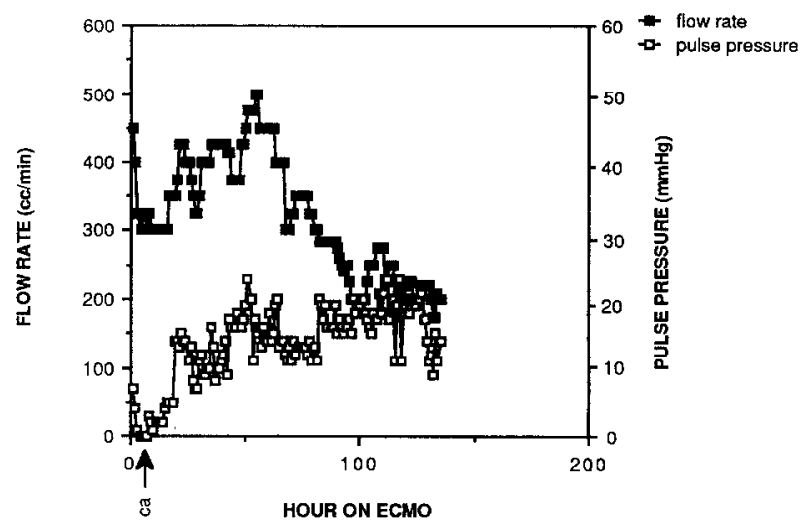

Fig 3. Pump flow rate and pulse pressure with time on ECMO. Cardiac dysfunction resolved without specific therapy after 8 hours. Ca, calcium.

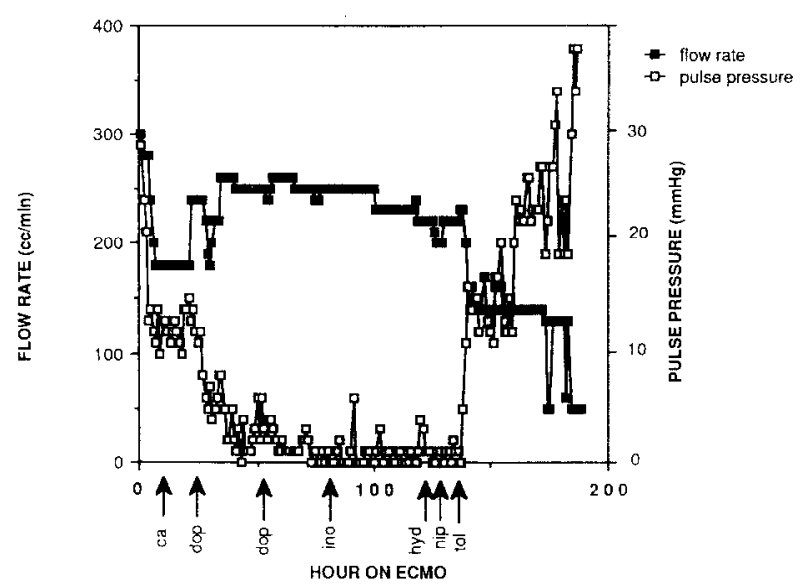

Fig 4. Pump flow rate and pulse pressure with time on ECMO. Pulse pressure appeared to decrease with dopamine (dop) and did not improve with hydralazine or amrinone (am) administration. Pulse pressure markedly improved with administration of tolazoline (tol) and nitroprusside (nit). Ca, calcium.

Electrolyte concentrations were normal with the exception of hypocalcemia $(8.1 \mathrm{mEq} / \mathrm{L})$ associated with hypoalbuminemia $(2.9 \mathrm{~g} / \mathrm{dL})$. The average creatinine at the start, as well as the creatinine and blood urea nitrogen (BUN) at the end of the ECMO course was elevated above the normal for the neonate population. However, none of the neonates developed oliguric renal failure. The creatinine phosphokinase with isoenzymes was evaluated in one child and was normal. Electrocardiography was performed in two children during periods of cardiac dysfunction. Both were normal.

Four of these 8 neonates had neurological sequelae that resulted in developmental delays and/or seizures. The other 4 were normal without any evidence of neurological or cardiac dysfunction.

\section{DISCUSSION}

The phenomenon of reversible ischemic cardiac dysfunction in neonates was noted in 1961 when Burnard and James recognized an association between birth asphyxia and subsequent radiographic and hemodynamic evidence of ventricular dilatation and myocardial dysfunction. ${ }^{5}$ Although $60 \%$ of these neonates died, the remainder survived with resolution of the cardiomyopathy. Transient cardiac dysfunction was also documented by Rowe and Hoffman in 1972 , when they observed three newborns with onset of cyanosis and congestive heart failure in the first 24 hours of life. 6 This cardiomyopathy resolved spontaneously between the second and fifth days of life and no cardiac sequelae were noted. Reversible cardiac dysfunction on prolonged cardiopulmonary bypass has been described in only one neonate to date. ${ }^{7}$ As with most of the neonates in the present study, the infant 
was profoundly hypotensive and hypoxic with a $\mathrm{PaO}_{2}$ of approximately $20 \mathrm{~mm} \mathrm{Hg}$ in the immediate preECMO period. Global dyskinesia of the ventricle developed after onset of bypass, which resolved over the ensuing 6 days.

Under normal circumstances, an infant on ECMO has adequate cardiac function to maintain at least a $>10 \mathrm{~mm} \mathrm{Hg}$ pulse pressure unless (1) cardiac tamponade or tension pneumothorax occur, or (2) flow rates approach those of total cardiopulmonary bypass. The former were ruled out in these newborns by echocardiogram and chest $\mathrm{x}$-ray and the latter through attempts at decreasing ECMO flow rate and fluid administration, which were not accompanied by the usual increase in native cardiac output and pulse pressure. In addition, the findings of myocardial dyskinesia noted on the echocardiogram of these newborns was different than the empty, but contracting, myocardium of other neonates studied while on total bypass. Therefore, it became evident that the primary problem was one of cardiac dysfunction after onset of bypass support.

This same group of children was at high risk for pre-ECMO cardiac hypoxia and ischemia. The average $\mathrm{PaO}_{2}$ immediately before onset of ECMO support was 26 , dramatically more hypoxic than the average $\mathrm{PaO}_{2}$ of 42 in the 715 neonatal ECMO cases reported to date and strikingly similar to the mean of $24 \mathrm{~mm} \mathrm{Hg}$ identified by Finley et $\mathrm{al}^{8}$ in a report on seven newborns with reversible ischemic cardiomyopathy. ${ }^{2}$ Additionally, $75 \%$ of the neonates had episodes of hypotension with or without bradycardia, all were receiving infusions of dopamine and/or dobutamine, $50 \%$ required cardiovascular support with epinephrine, and $25 \%$ underwent cardiac arrest in the immediate pre-ECMO period. The average OI was almost double the value that identifies the $80 \%$ mortality group in this nursery. Overall, this describes a group of newborns who were much more hypoxic and compromised from a cardiovascular point of view than the average ECMO child. Indeed, $50 \%$ of these children had signs of renal insufficiency (elevations in both the BUN and creatinine at conclusion of the ECMO course) and 50\% of these newborns demonstrated neurological deficits in their follow-up. In routine ECMO cases, the incidence of insufficiency/ deficits in these organ systems is only $10 \%$ and $20 \%$ to $30 \%$, respectively. ${ }^{3}$ At least $75 \%$ of these newborns demonstrated clear signs of pre-ECMO cardiac dysfunction, and, apparently, other organ systems were affected as well.

Once on bypass support, cardiac dysfunction persisted even though adequate myocardial oxygen supply was reestablished. The primary problem was one of persistent myocardial dysfunction possibly exacerbated by (1) the increased afterload presented to the heart by the ECMO perfusion, and/or (2) preload reduction during venoarterial bypass." In all instances, the dysfunction was self-limiting, lasting in the range of hours to days and usually resolving without specific therapy. Myocardial afterload reduction with hydralazine or tolazoline in three prolonged cases did appear to be beneficial.

The reversible nature of some forms of cardiac dysfunction after cardiac operation has been demonstrated in adults and children supported with ECMO or with ventricular-assist devices. ${ }^{10-13}$ Approximately $50 \%$ of these patients, mostly in the pediatric age group, demonstrated improvement in cardiac function over a period of days. ${ }^{13}$ It is likely that intraoperative myocardial ischemia is the basis for this reversible postoperative cardiac dysfunction. In 1982, Braunwald and Kloner promoted the concept of the "stunned myocardium," which described the postischemic heart with prolonged but transient and reversible dysfunction. ${ }^{4}$ Studies of acute canine coronary occlusion for periods up to 15 minutes were not associated with myocardial infarction, but resulted in transient dysfunction of the ischemic myocardium for several days. ${ }^{14}$ Myocardial adenosine triphosphate (ATP) concentrations have been shown to remain reduced and ultrastructural abnormalities observed in these myocardial cells for up to 3 days following reperfusion. ${ }^{15}$ These findings, along with ventricular function, resolved by 7 days. It appears that the ischemic myocardium may be stunned and remain transiently dysfunctional for a period of days until myocardial intracellular ATP levels are repleted. ${ }^{16}$ This phenomenon has been documented in adults after coronary occlusion and early reperfusion with thrombolysis or revascularization. ${ }^{17-19}$ Resolution of myocardial dysfunction over a period of days has also been demonstrated after coronary vasospasm in the adult. ${ }^{20}$ As mentioned previously, transient reversible myocardial dysfunction of the newborn has been documented. ${ }^{5.8}$

Presented here are eight neonates who developed similar transient, reversible episodes of myocardial dysfunction after periods of exposure to profound levels of total-body hypoxia and/or hypoperfusion. The decreased contractility appeared to be global in nature, which is typical for ischemia-induced dysfunction in the neonatal heart. ${ }^{20}$ Serial creatinine phosphokinase in one newborn and electrocardiographic evaluation in two did not show any evidence of myocardial infarction or ischemia. This is consistent with the findings in some patients with myocardial stun, although numerous cases of reversible dysfunc- 
tion have been accompanied by transient $Q$ waves and flattened $T$ waves suggestive of ischemia. ${ }^{6.818,20}$ With ECMO support, the cardiac dysfunction in this study resolved in all eight neonates. Most resolved without any specific therapy over the ensuing 24 hours. Those neonates with evidence of cardiac dysfunction for longer periods demonstrated improvement with systemic afterload reduction. Therefore, the patient with postischemic cardiac dysfunction on ECMO should be initially watched with the expecta- tion that myocardial contractility will improve over the ensuing 24 hours. After that time period, trials of afterload reduction with hydralazine or tolazoline may be beneficial.

Overall, these eight patients, along with the successful postcardiac procedure ECMO cases mentioned previously, serve to highlight the potentially reversible nature of postoperative or postischemic cardiac dysfunction, especially in the neonatal and pediatric population.

\section{REFERENCES}

1. Bartlett RH, Gazzaniga A, Toomasian J, et al: Extracorporeal membrane oxygenation (ECMO) in neonatal respiratory failure: 100 cases. Ann Surg 204:236-245, 1986

2. Hirschl RB, Bartlett RH: Extracorporeal membrane oxygenation support in cardiorespiratory failure. Adv Surg 21:189-212, 1987

3. Toomasian JM, Snedecor SM, Cornell RG, et al: National experience with extracorporeal membrane oxygenation for newborn respiratory failure. Trans Am Soc Artif Intern Org 34:140147, 1988

4. Braunwald E, Kloner RA: The stunned myocardium: Prolonged, postischemic ventriculart dysfunction. Circulation 66:11461149,1982

5. Burnard E, James L: Failure of the heart after undue asphyxia at birth. Pediatrics 28:545-565, 1961

6. Rowe RD, Hoffman T: Transient myocardial ischemia of the newborn infant: A form of severe cardiorespiratory distress in full-term infants. J Pediatr 81:243-250, 1972

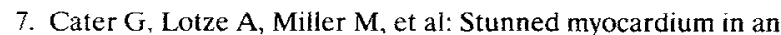
infant treated with extracorporeal membrane oxygenation. J Pediatr Surg 23:1011-1013, 1988

8. Finley JP, Howman-Giles RB, Gilday DL, et al: Transient myocardial ischemia of the newborn infant demonstrated by thallium myocardial imaging. Fetal Neonat Med 94:263-270, 1979

9. Kresh JY, Kerkhor PL, Goldman SM, et al: Heart-mechanical assist device interaction. Trans Am Soc Artif Intern Org 32:437445,1986

10. Bartlett RH, Gazzaniga AB, Fong SW, et al: Extracorporeal membrane oxygenator support for cardiopulmonary failure. J Thorac Cardiovasc Surg 73:375-386, 1977

11. Pierce WS, Grant VSP, Myers JL, et al: Ventricular-assist pumping in patients with cardiogenic shock after cardiac operations. N Engl J Med 305:1606-1610, 1981

12. Pennington DG, Merjavy JP, Codd JE, et al: Extracorporeal membrane oxygenation for patients with cardiogenic shock. Circulation 70:130-137, 1984 (suppl I)

13. Kanter KR, Pennington DG, Weber TR, et al: Extracorporeal membrane oxygenation for postoperative cardiac support in children. J Thorac Cardiovasc Surg 93:27-35, 1987

14. Heyndrickx GR, Millard RW, McRitchie FJ, et al: Regional myocarcial function and electrophysiological allerations after brief coronary artery occlusion in conscious dogs. $\mathbf{J}$ Clin Invest 56:978985, 1975

15. DeBoer FWV, Ingwall JS, Kloner RA, et al: Prolonged derangements of canine myocardial purine metabolism after a brief coronary artery occlusion not associated with anatomic evidence of necrosis. Proc Natl Acad Sci USA 77:5471-5475, 1980

16. Reimer, KA, Hill ML, Jennings RB: Prolonged depletion of ATP and of the adenine nucleotide pool due to delayed resynthesis of adenine nucleotides following reversible myocardial ischemic injury in dogs. J Mol Cell Cardiol 13:229-239, 1981

17. Ganz W, Geft I, Maddahi J, et al: Nonsurgical reperfusion in evolving myocardial infarction. J Am Coll Cardiol 5:1247-1253, 1983

18. Bateman TM, Czer LSC, Gray RJ, et al: Transient pathologic $Q$ waves during acute ischemic events: An electrocardiographic correlate of stunned but viable myocardium. Am Heart $\mathbf{J}$ 6:1421-1426, 1983

19. Kloner RA, Ellis SG, Carlson NV, et al: Coronary reperfusion for treatment of acute myocardial infarction: Post-ischemic ventricular dysfunction. Cardiology 70:233-246, 198.3

20. Mathias P, Kerin NZ, Blevins RD, et al: Coronary vasospasm as a cause of stunned myocardium. Am Heart J 113:383-385. 1987 